\title{
EXIT Analysis for 5G NR Demapper and Further Enhancements
}

\author{
Fadli Afandi Putra ${ }^{1}$, Khoirul Anwar ${ }^{2}$, Levy Olivia Nur ${ }^{3}$ \\ \{fadliafandiputra@student., anwarkhoirul@, levyolivia@\} \\ telkomuniversity.ac.id \\ Center for Advanced Wireless Technologies (AdWiTech), \\ School of Electrical Engineering, Telkom University
}

\begin{abstract}
The fifth telecommunication generation (5G) New Radio (NR) demands excellent modulation to enhance spectral efficiency, where symbol-to-bit mapping rule in constellation diagram must be seriously considered. 5G NR uses Gray modulation that requires high performance coding, where computational complexity is usually high. This Paper proposes new 5G NR non-Gray modulation having capability of iterative decoding such that simple decoding can be applied. The iterations between demapper and decoder are utilized to update Log Likelihood Ratio (LLR) value of each transmitted bit to minimize error, where non-Gray modulation has better performance compared to Gray modulation. We propose new non-Gray modulation using Extrinsic Information Transfer (EXIT) chart to design matching between modulation and coding. We evaluate them using a series of computer simulations and design a mapper that matches the simplest decoding scheme, i.e., repetition codes. The results are expected to be better than the original 5G NR modulations.
\end{abstract}

Keywords: 5G New Radio, iterative demapping, non-Gray, modulation, and EXIT chart.

\section{Introduction}

The Third Generation Partnership Project (3GPP) is lately in the process of specifying the fifth telecommunication generation (5G) New Radio (NR). Mobile broadband (MBB) will become important and provide the need for higher system capacity, better coverage, and higher data rates up to $20 \mathrm{Gbps}[10]$. However, the purpose of $5 \mathrm{G}$ is more than that. One example is massive machine-type communication (mMTC), sometimes also called as the Internet of Things (IoT), where key challenges are to enable very low device cost and energy consumption, and handle very large numbers of devices [3]. Another example is ultra-reliable low-latency communication (URLLC), providing data delivery with unprecedented reliability in combination with very low latency, for example, targeting critical industry applications [9]. The eMBB, mMTC, and URLLC are there main concepts of 5G NR according to the IMT (International Mobile Telecommunications)-2020 standard as shown in Figureure 1. 


\section{Enhanced mobile broadband}

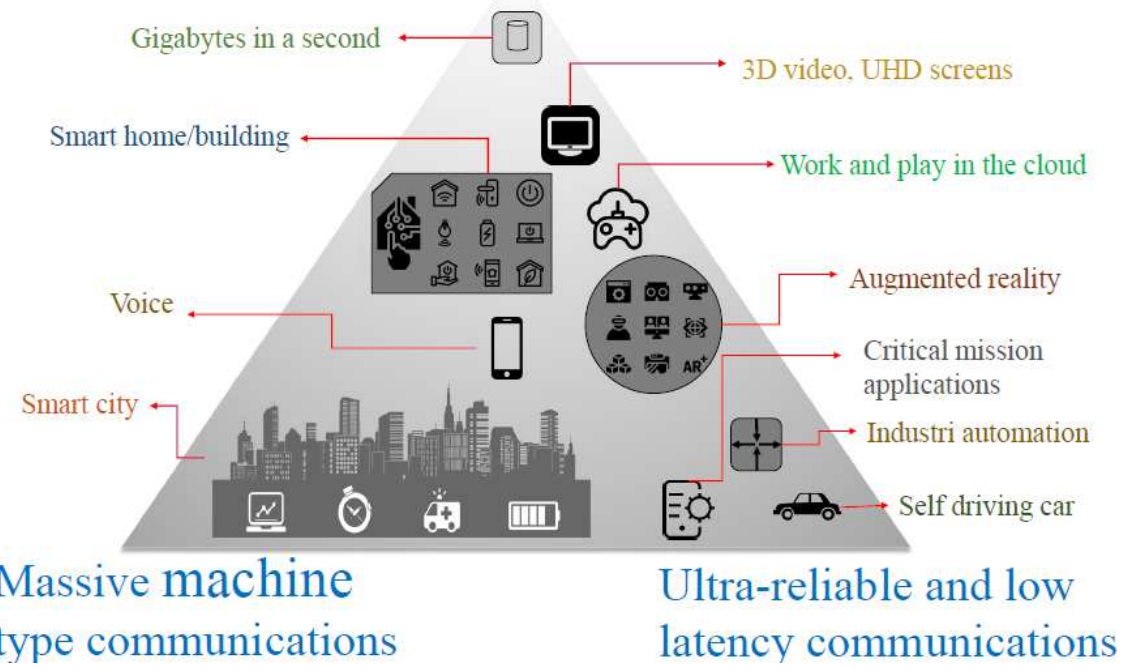

Figure. 1. 5G NR concept according to IMT standard.

3GPP has released a standard TS.38.211 that includes saveral modulations for 5G NR such as BPSK, QPSK, 16-QAM, 64-QAM, and 256-QAM. These modulations is to support the 5G NR that wants to increase data rates 10 until 100 times higher than the previous telecommunication generation, long term evolution (LTE).

This Paper proposes a technique to modify the receiver side which is on demapper. The proposed demapper has capability of iterative decoding. Demapper is helped by repetition decoder for detecting the bit. In fact, the iterations between demapper and decoder make the system more complex. In another side, it can decrease bit error probability significantly. It is because demapper and decoder update the log-likelihood ratio (LLR) value in each iteration.

Iterations process between demapper and decoder is evaluated using extrinsic transfer information (EXIT) chart to know the iterative behaviour of LLR value on these two components. The EXIT chart pioneered by Stephan ten Brink is powerful technique which is used to analyze the behavior of iterative so-called turbo techniques[5].

There are two types of modulation, Gray and non-Gray. Gray modulation is mapper that has all symbols, which are near each other, have one bit of hamming distance. Meanwhile, nonGray modulation is a mapper where its two nearest symbols have more that one bit of hamming distance. The characteristic of 5G NR mapper is unidentified. This Paper identify the mapper by using EXIT chart. This identification is important because the proposed demapper only works while using non-Gray modulation. The modification on 5G NR mapper is needed if it is identified as Gray modulation. This Paper identify QPSK and 16-QAM 5G NR so that proposed demapper can be applied. 


\section{Review on Model Sistem}

This Paper focuses to modify mapper and demapper. This Paper proposes demapper which capability of iterative decoding, where the demapper gets a fed back LLR from the decoder. Figureure 2 shows system model. Repetition codes $C$ add parity bits into information bit $b$. The sequence bits of parity bits and information bits is called codeword $c$. The sequence of codeword is interleaved as a permutation value of codeword length by interleaver $\Pi$. Mapper $M$ maps the codeword into complex-value symbol before it is transmitted to channel. Demapper $M^{-1}$ calculates LLR value of received bit. $A C C^{-1}$ removes dopped LLR. LLR value is updated in each iteration between demapper and decoder.

\subsection{Encoder}

Channel coding is commonly known as encoder $C$ or decoder $D$. It takes a role for encrypting information bit $b$ to againts distortion effect that is produced by noise $n$. A channel coding encapsulates information bit using redudancy bits [8]. The redudancy bit is called as a parity bit. The sequence bit that contains information bit and parity is called as codeword. Proportion of information bit $k$ and parity bit $(n-k)$ can be defined as

$$
R=\frac{k}{n}
$$

The codeword $c$ is defined as

$$
c=m G
$$

where the repetition codes generator matrix is

$$
G=\left[\begin{array}{lll}
1 & 1 & 1
\end{array}\right]
$$

A sequence $b$ information bit which is transmitted in binary formed is encoded by regular repetition codes. Each information bit repeat $n$ times. 


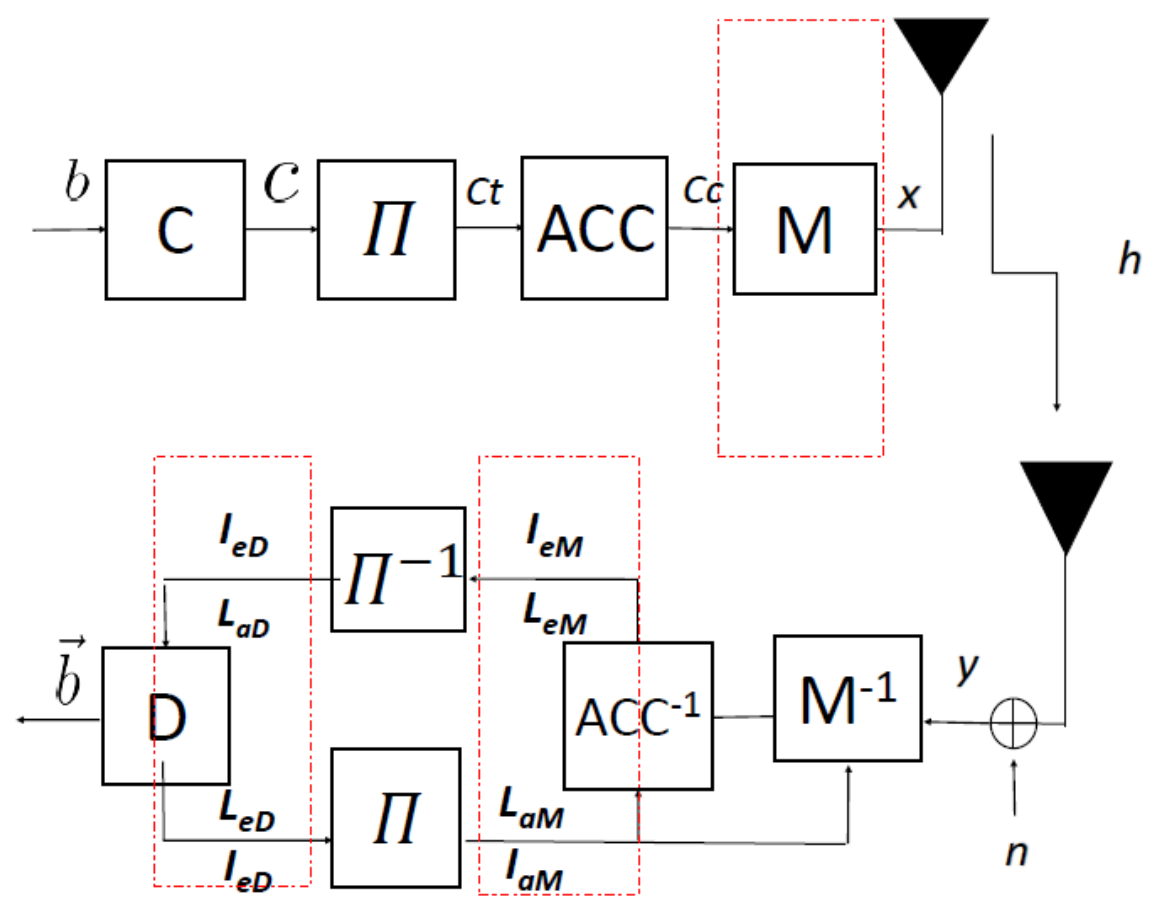

Figure. 2. System Model.

\subsection{Modulation}

3GPP has released TS38.211 standard that informs about the modulation which is used by $5 \mathrm{G} \mathrm{NR}$ in the future. This modulation use the binary, 0 or 1 , as an input for its formula. A sequence bit is converted to be a complex-value symbol [2]. The number of sequence bits that can be converted is as the modulation use. This following subsection explain how the sequence bits is converted to be the complex-value symbol.

\subsubsection{Quadrature Phase Shift Keying}

While the system use the QPSK, a pair bit $c(i), c(i+1)$ is mapped to a complex-value symbol as this following formula [1]

$$
x=\frac{1}{\sqrt{2}}[(1-2 c(i))+j(1-2 c(i+1))],
$$

the 4 complex-value symbols are plotted to the constellation diagram as shown in Figureure 3(a). 


\subsubsection{6-Quadrature Amplitude Modulation}

The 16 of quadruplets bits; $c(i), c(i+1), c(i+2), c(i+3)$ are mapped to 16 complexvalue symbols as this following formula[1]

$x=\frac{1}{\sqrt{10}}\{(1-2 c(i))[2-(1-2 c(i+2))]+j(1-2 c(i+1))[2-(1-2 c(i+3))]\}$,

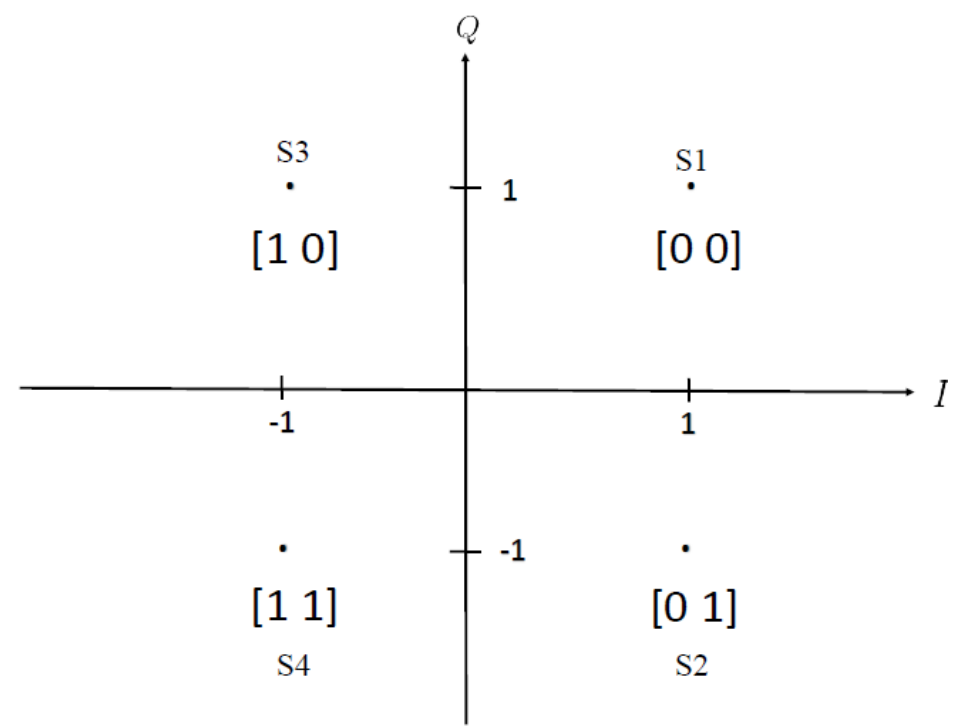

(a) 


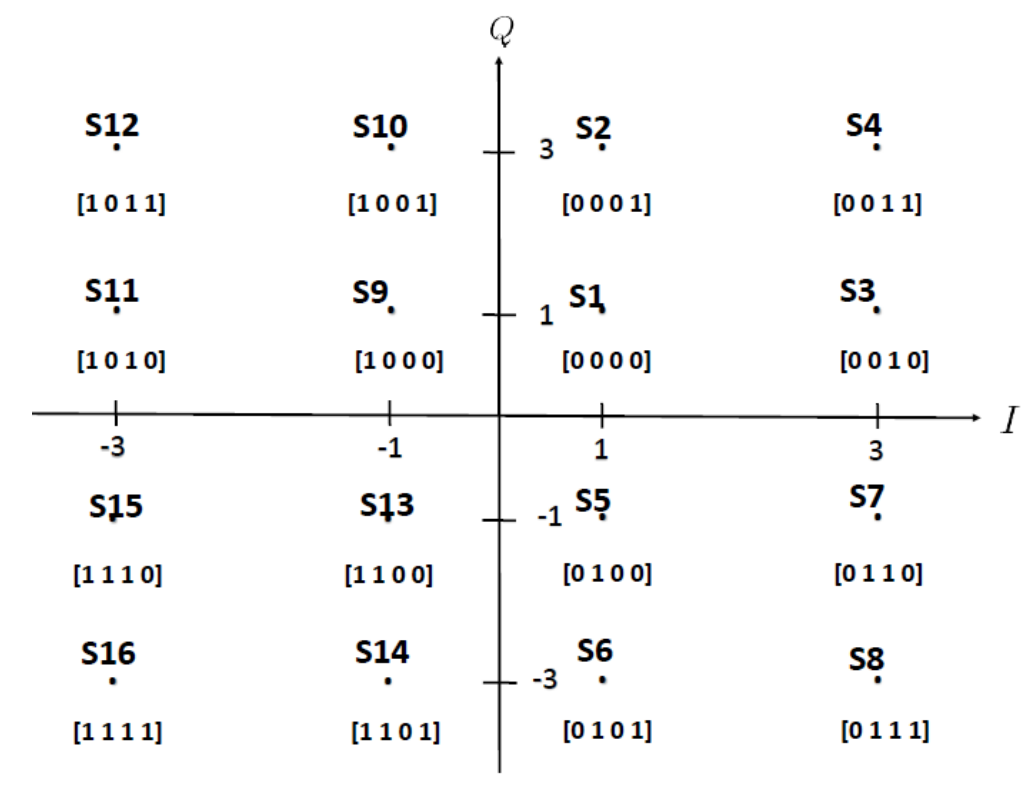

(b)

Figure. 3. QPSK 5G NR constellation diagram(a); 16-QAM 5G NR constellation diagram(b).

the 16 complex-value symbols are plotted to the constellation diagram as shown in Figureure 3. Based on Figureure 3, QPSK and 16-QAM is Gray modulation because the symbols have hamming distance one bit to each other.

Hamming distance is a number of bits of two symbols which are not matched. Symbol A with bits [10] and symbol B has bits [11]. Symbol A and symbol B have hamming distance of one bit because their second bit are different.

\subsection{Channel}

On the AWGN channel, the noise source is in the form of thermal noise on the electronic components at the receiver. "Additive" means that thermal noise is added to the received signal, while "White Gaussian noise" means that the power of the existing noise has the same spectral density at all frequencies. This system is simulated in single path $h$ AWGN, the transmitted signal can be determined as

$$
y=h * n+\text { noise. }
$$

\subsection{Demodulation}

Demodulation is a process to calculates LLR value of each bit that is brought by received signal [7]. The component to do it is demodulator or demapper.

With the help of a priori information $L_{a, M}$ that is provided by regular repetition codes decoder, the demapper calculates the extrinsic LLR $L_{e, M}[4]$ of the bit $q[v]$ which is brought by the received signal $r . L_{e, M}$ can be determined as 


$$
\begin{aligned}
& L_{e, M}(q[v])=\ln \frac{P(q[v]=0 \mid r)}{P(q[v]=1 \mid r)} \\
& =\ln \frac{\sum_{s \epsilon s_{0}} \exp -\frac{|r-h s|}{2 * \sigma^{2} \mid} \prod_{w=1, w \neq v}^{l} \exp -q[w] L_{a, M}(q[w])}{\sum_{s \epsilon s_{1}} \exp -\frac{|r-h s|}{2 * \sigma^{2}} \prod_{w=1, w \neq v}^{l} \exp -q[w] L_{a, M}(q[w])},
\end{aligned}
$$

where $\mathrm{s} 0$, s1 refers to the set of labels having bit $\mathrm{q}[\mathrm{v}]$ being 0 and 1 , respectively. $\operatorname{La}, M(q[v])$ is the a priori LLR fed back from the repetition decoder corresponding to the vth bit position in the label $\mathrm{q}$ allocated to the signal point $\mathrm{s}$

\subsection{Decoder}

Repetition decoder gives a feedback to the demapper. $L_{e, M}$ from the demapper is decoded, then the result is $L_{a, M}$ that becomes a feedback for the demapper. Demapper and repetition decoder update the LLR so that the bit error can be reduced.

\section{Modification on 5G NR Mapper}

In this section, we focus on how we modify the 5G NR Mapper so that it can be suitable for proposed demapper that has capability of iterative decoding. We modify two 5G NR mapper, QPSK and 16-QAM.

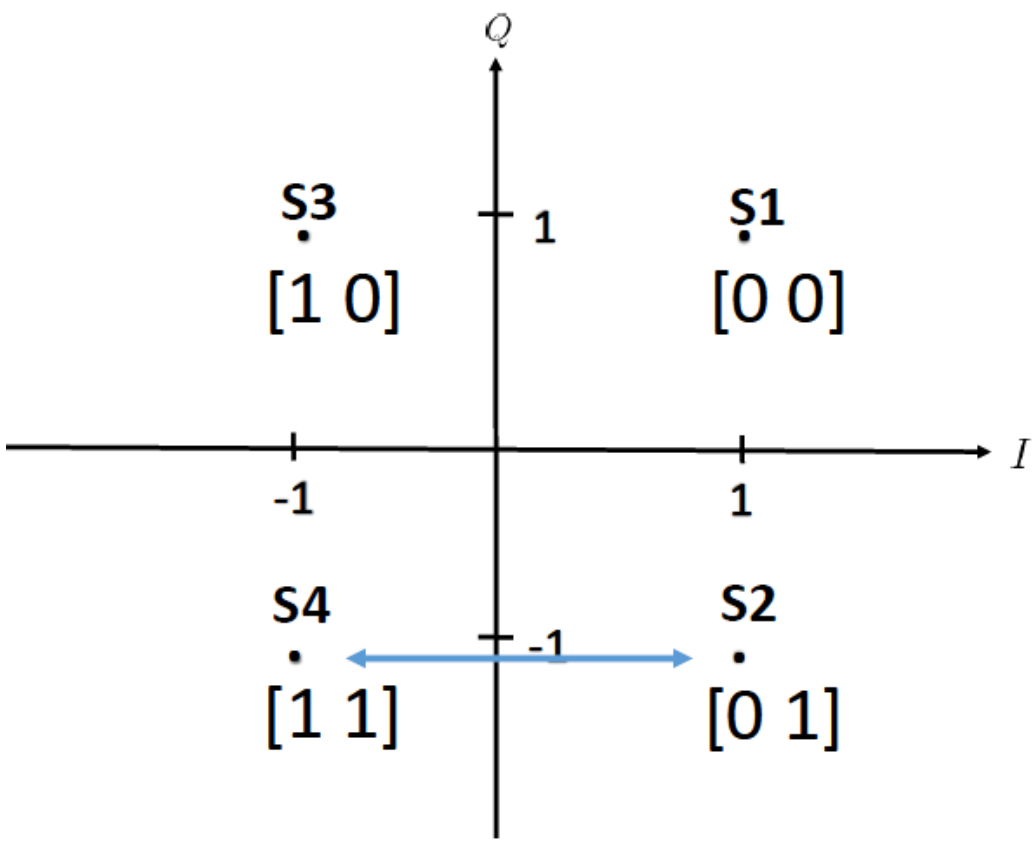


Figure. 4. Modification on 5G NR mapper.

\subsection{QPSK}

As shown in Figureure 3(a), QPSK 5G NR mapper is Gray scheme because hamming distance of each symbol is 1 bit. By swapping symbol 2 (S2) and symbol 4 (S4), S1 and S4 have 2 bit of hamming distance, S2 and S3 also have 2 bit of hamming distance. So that the modulation that its symbol have more than 1 bit hamming distance to the other symbol can determine as non-Gray scheme modulation. The modification of QPSK 5G NR mapper can be shown in Figureure 4. For the validation of that non-Gray modulation, we use EXIT chart. We explain the way we validate it is on the next section.

\subsection{6-QAM}

The 16-QAM 5G NR mapper is shown in Figureure 3(b). Based on constelation we know that 16-QAM 5G NR is also Gray scheme modulation as the explanation in the section 2 . We have several steps for changing the 16-QAM 5G NR mapper to be non-Gray scheme modulation. The Figureure 5 are steps how we modify 16-QAM 5G NR to be nonGray scheme.

- The first step is shown in Figureure 5(a). Four symbols in quadran II is rotated $45^{\circ}$ counter clockwise.

- Then, symbol 2 (S2) and symbol 4 (S3) is swapped each other. The next pair symbols that are swapped each other are S10 and S9; S14 and S13; the last is S7 and S5. It can be shown in Figureure 5(b).

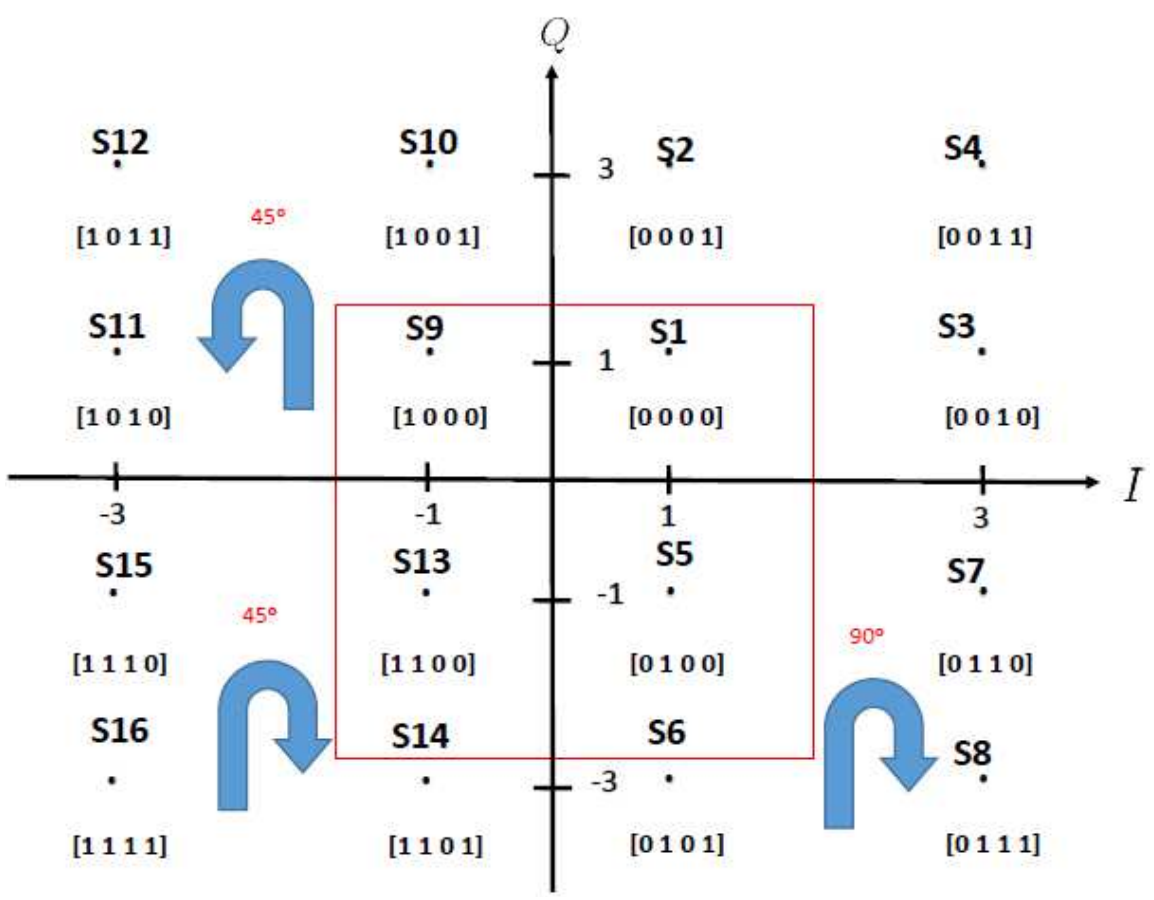


(a)

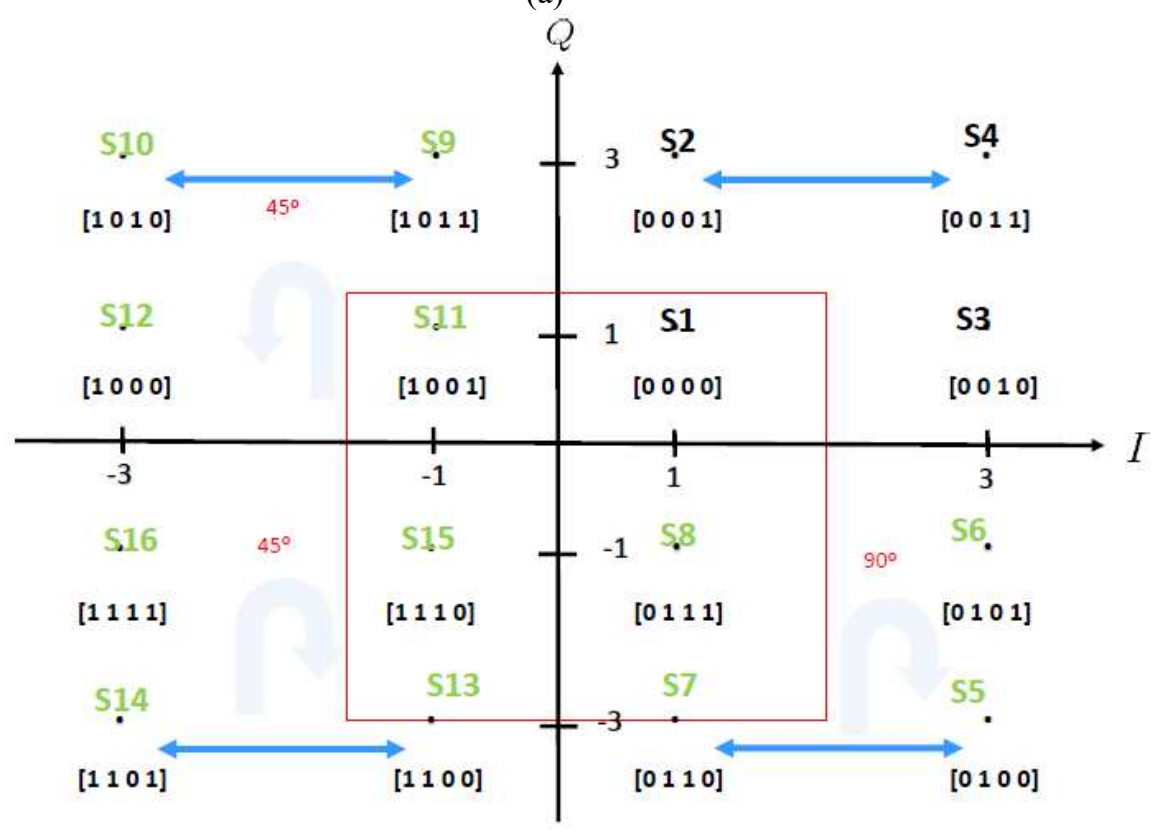

(b)

Figure. 5. Step 1 (a) and step 2 (b) of modification on 16-QAM 5G NR mapper.

\section{EXIT Chart Properties}

EXIT chart of demapper is determined by $L a$ and $L e$ value on demapper. $L a$ is LLR which gets into demapper and being multiplier to calculate $L e$. $L a$ can be determined as

$$
\begin{aligned}
& \sigma=\frac{\left(-1.0 / 0.3037 * \log \left(1.0-M I^{(1.0 / 1.1064)}\right)\right.}{\left.\log (2.0))^{1.0 /(2.0 * 0.8935)}\right)} \\
& L a=\frac{\operatorname{sigma}^{2} *(1-2 * b i t s)}{2}+\operatorname{randn}(1, \text { length }(\text { bits }) * \sigma,
\end{aligned}
$$

where $M I$ atau $I A$ is mutual information apriori and its value is set as 0:0,1:1. IE values can be determined by using histogram calculation between $L e$ and codeword. Meanwhile, EXIT chart of repetitions codes can be determined as

$$
I E=1-(1-I A)^{(d v-1)},
$$

where $d v$ is $\mathrm{n}$ repeat times of repetition codes 


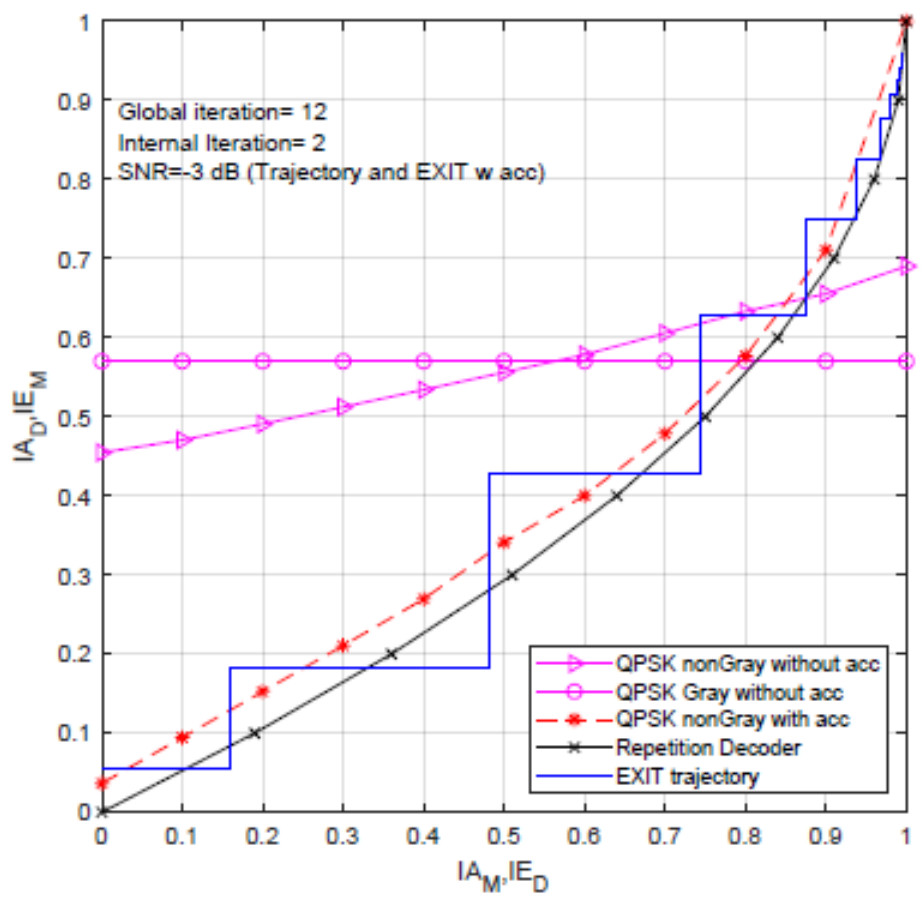

Figure. 6. EXIT chart of QPSK for different modulation scheme. 


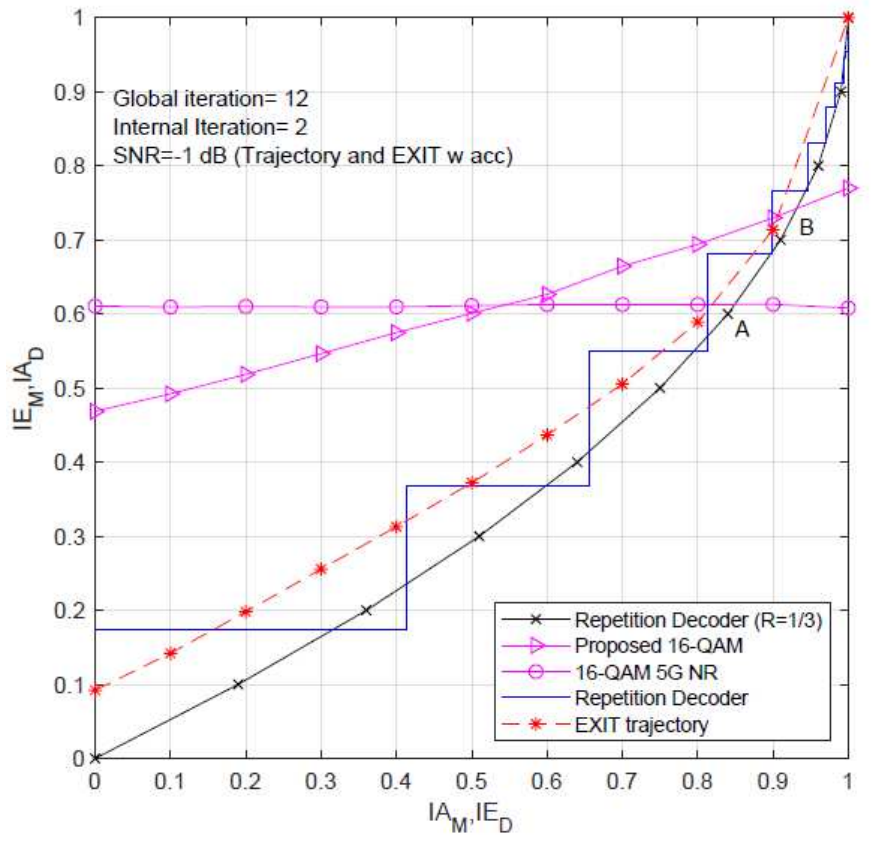

Figure. 7. EXIT chart of 16-QAM for different modulation scheme.

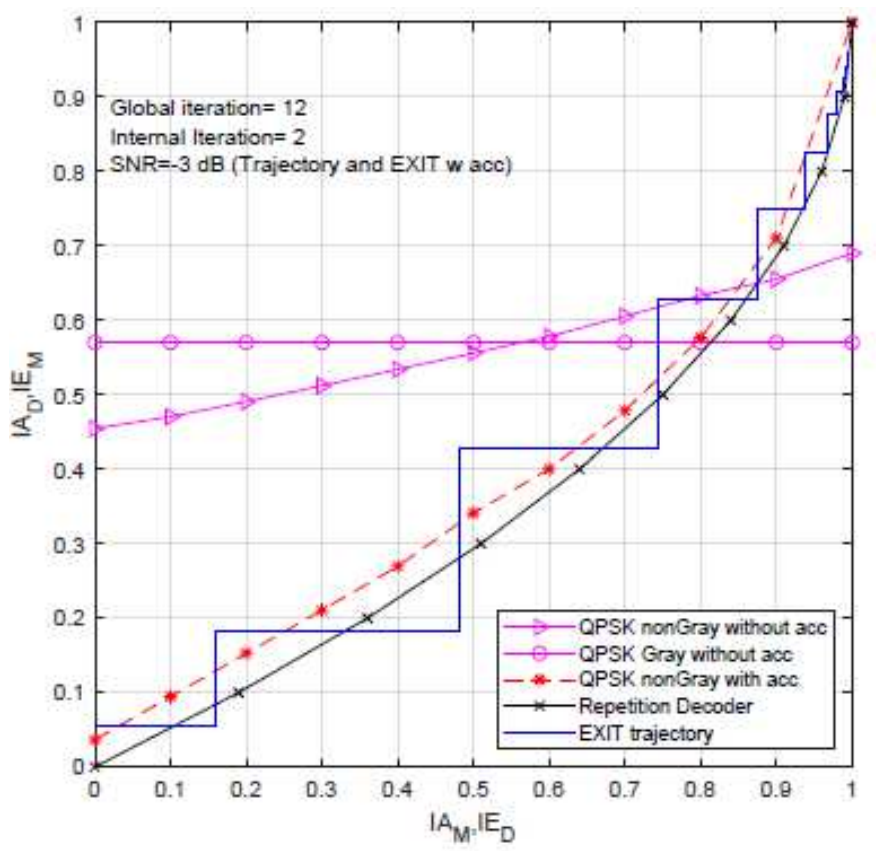


Figure. 8. BER performance of QPSK on AWGN channel.

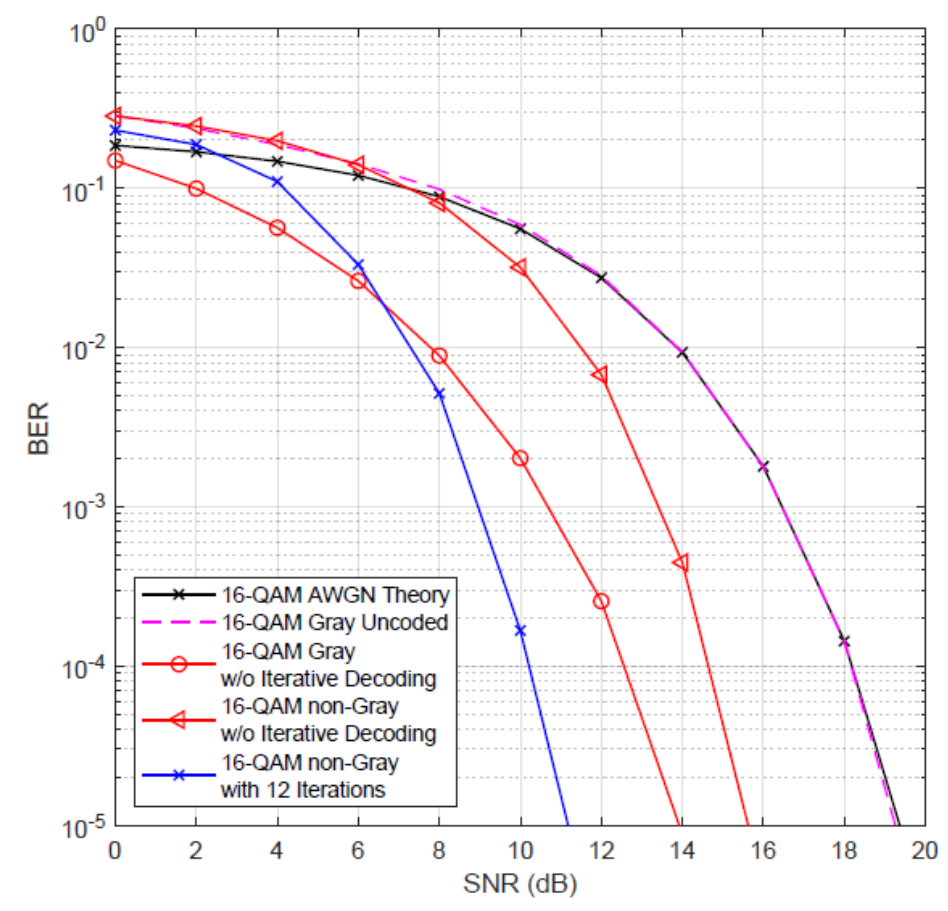

Figure. 9. BER performance of 16-QAM on AWGN channel.

\section{EXIT Chart Analysis}

QPSK and 16-QAM 5G NR are Gray modulation based on the diagram constellation. It causes they are not suitable for proposed demapper that has capability of iterative decoding. EXIT chart verify the characteristic of original and modified 5G NR mapper. Beside that, the EXIT chart is to examines the performance of proposed demapper. This Paper is to find out the closest gap between EXIT chart of demapper and decoder.

EXIT chart which has flat form indicates the mapper is Gray, while the EXIT chart which has increasing $I E$ value is non-Gray. The flat form means that the iterations do not work. The LLR value of Gray modulation cannot be updated because the hamming distance of two nearest symbols are one bit. The increasing form means that the iterations is success to update the LLR so that the $I E$ value increased.

EXIT chart of QPSK and 16-QAM is shown in Figureure 6 and 7 respectively. The purple curve with a round sign is EXIT chart of demapper with 5G NR mapper, while the purple curve with a triangle sign is EXIT chart of demapper with modified mapper. EXIT chart of demapper 
with 5G NR mapper shows the flat form, while EXIT chart of demapper with modified mapper shows the increasing form. The red dashed curve is EXIT chart of proposed demapper using accumulator. It has the best performance because it has smallest gap with EXIT chart of repetition decoder which is shown by black curve with cross sign.

BER performance of QPSK and 16-QAM is shown in Figureure 8 and 9 respectively. BER performance is to validate the performance that is shown by EXIT chart. The black curve with cross sign is theoretical BER [6] that is defined as

$$
\begin{aligned}
& B E R_{Q P S K_{-} A W G N}\left(E_{b} / N_{0}\right)=\frac{1}{2} \operatorname{erfc}\left(\sqrt{E_{b} / N_{0}}\right), \\
& B E R_{256 Q A M_{-} A W G N}\left(E_{b} / N_{0}\right)=\frac{3}{8} \operatorname{erfc}\left(\sqrt{\frac{2}{5} E_{b} / N_{0}}\right) \\
& -\frac{9}{64} \operatorname{erfc}^{2}\left(\sqrt{\frac{2}{5} E_{b} / N_{0}}\right) .
\end{aligned}
$$

The purple dashed curve is BER performance of the system without channel coding. BER performance of demapper without iterative decoding using Gray mapper and non-Gray mapper are shown respectively as red curve with a round sign and red curve with triangle sign. Gray has better performance than non-Gray because it has the balanced hamming distance. BER performance of demapper with iterative decoding is depicted by the blue curve with star sign. Demapper with iterative decoding needs 12 iterations to achieve best performance based on EXIT trajectory. Demapper with iterative decoding has more efficient power of $3 d B$ at $10^{-5}$ than demapper without iterative decoding has.

\section{Conclusion}

This Paper has proposed modification of QPSK and 16-QAM 5G NR mapper. This modification is to make it suitable for demapper with iterative decoding so that the system can improve its performance.

According to the simulation, mapper QPSK and 16-QAM 5G NR are Gray modulation. It is shown by their EXIT chart form which is flat. Gray modulation is not suitable for demapper with iterative decoding because the iterations do not work in this modulation scheme.

We modify mapper QPSK and 16-QAM 5G NR to be non-Gray modulation. The EXIT chart after this modification show increasing trend. It means that the iteration for the system works. However, the gap between demapper and decoder EXIT chart is still wide so that we add the accumulator. The result show that the Gap of these two EXIT chart become smaller than before. The smaller gap between two EXIT chart have, the better performance the system can achieve. According to the result, the demapper with iterative decoding capability has better performance than the non-iterative decoding system. The performance of the demapper the iterative system reach about $3 \mathrm{~dB}$ more efficient than the non-Iterative system at BER $10^{-} 5$. 


\section{References}

[1] J. G. Andrews et al., "What will 5G be?," IEEE J. Sel. Areas Commun., vol. 32, no. 6, pp. 1065 1082, 2014.

[2] TSGR, "TS 138211 - V15.3.0 - 5G; NR; Physical channels and modulation (3GPP TS 38.211 version 15.3.0 Release 15)," vol. 0, 2018.

[3] A. F. Molisch, Wireless Communications" (Molisch, A.; 2011) [Book review], vol. 19, no. 1. 2012.

[4] K. Anwar and T. Matsumoto, "Very simple BICM-ID using repetition code and extended mapping with doped accumulator," Wirel. Pers. Commun., vol. 67, no. 3, pp. 573-584, 2012.

[5] H. Harada and R. Prasad, "Simulation and Software Radio for Mobile Communications." p. 467, 2002.

[6] M. Abdullahi and P. Xiao, "Performance analysis and soft demapping for coded MIMO-OFDM systems," Proc. Int. Symp. Wirel. Commun. Syst., vol. 2016-October, no. December 2017, pp. 242-246, 2016.

[7] T. K. Moon, "Error Correction Coding Mathematical Methods and Algorithms," J. Chem. Inf. Model., vol. 53, no. 9, pp. 1689-1699, 2013.

[8] A. Osseiran, V. Braun, and T. Hidekazu, "The foundation of the mobile and wireless communications system for 2020 and beyond," Proc. IEEE Veh. Tech. ..., pp. 1-5, 2013.

[9] E. Dahlman and S. Parkvall, "NR - The new 5G radio-access technology," IEEE Veh. Technol. Conf., vol. 2018-June, pp. 1-6, 2018.

[10] J. Hagenauer, “The \{EXIT\} chart - Introduction to Extrinsic Information Transfer," Proc. 12th Eur. Signal Process. Conf., pp. 1541-1548, 2004. 